\title{
Tecnología de la Información y Comunicación en estudiantes del nivel primario en el marco de la educación inclusiva en un Centro de Educación Básica Especial
}

\section{Information and Communication Technology in Primary School Students within the Framework of Inclusive Education at a Special Basic Education Center}

\author{
Ricardo Iván Vértiz-Osores \\ Pontificia Universidad Católica del Perú, Lima, Perú. \\ ORCID: https://orcid.org/0000-0003-1223-2784 \\ Segundo Pérez-Saavedra \\ Pontificia Universidad Católica del Perú, Lima, Perú. \\ ORCID: https://orcid.org/0000-0002-2366-6724 \\ Miguel Ángel Faustino-Sánchez \\ Universidad Nacional Mayor de San Marcos, Lima, Perú. \\ ORCID: https://orcid.org/0000-0002-5445-4281 \\ Jacinto Joaquín Vértiz-Osores* \\ Universidad Peruana Cayetano Heredia, Lima, Perú \\ ORCID: https://orcid.org/0000-0002-7606-476X \\ Lineth Alain \\ Universidad Tecnológica de Panamá, Panamá., Panamá
}

Recibido 30-08-18 Revisado 15-09-18 Aprobado 08-12-18 En línea 12-12-18

*Correspondencia

Email: jacinto.vertiz.o@upch.pe

\begin{tabular}{l} 
Citar como: \\
\hline Vértiz-Osores, R., Pérez-Saavedra, S., Faustino-Sánchez, \\
M., Vértiz-Osores, J., \& Alain, L. (2019). Tecnología de la \\
Información y Comunicación en estudiantes del nivel \\
primario en el marco de la educación inclusiva en un Centro \\
de Educación Básica Especial. Propósitos y \\
$\begin{array}{l}\text { Representaciones, } \quad 7(1), \quad 146-164 . \quad \text { Doi: } \\
\text { http://dx.doi.org/10.20511/pyr2019.v7n1.266 }\end{array}$
\end{tabular}

(C) Universidad San Ignacio de Loyola, Vicerrectorado de Investigación, 2019.

(cc) $\mathrm{BY}-\mathrm{NC}-\mathrm{NO}$

Este artículo se distribuye bajo licencia CCBY-NC-ND 4.0 Internacional.http://creativecommons.org/licenses/by-nc-nd/4.0/). 


\section{Resumen}

Esta investigación tiene como objetivo conocer el impacto de las tecnologías de la información y la comunicación (TIC), como mecanismo innovador aplicado en experiencias curriculares docentes, para el desarrollo de habilidades cognitivas y socio emocionales de estudiantes entre 08 a 13 años del nivel primaria que presentaron discapacidad para hablar o moverse dentro del contexto de un Centros de Educación Básica Especial (CEBE). Fue una investigación cualitativa, sustantiva. Se usó la herramienta tecnológica Plaphoons (software libre creado para necesidades educativas especiales). Se realizó el análisis de contenidos de las intenciones, percepciones, observación, registro de datos mediante la triangulación de datos y categorización de contenidos para la emisión de resultados. Se concluyó que el uso de esta TIC potenció las capacidades de esos estudiantes, constatando mejoras cognitivas para el aprendizaje de los tópicos de lógico matemática, comunicación y ciencias sociales. Asimismo, se constató que esta herramienta permite un mejor y mayor desarrollo de sus habilidades sociales.

Palabras clave: TIC; Educación inclusiva; cognitivas; socioemocionales.

\section{Summary}

This research aims to know the impact of information and communication technologies (ICT), as an innovative mechanism applied in curricular teaching experiences, for the development of cognitive and socio-emotional skills of students aged between 08 to 13 years from primary school who had disabilities to speak or move within the context of a Special Basic Education Center (CEBE). It was a qualitative, substantive research. The Plaphoons (free software created for special education needs) technological tool was used. The analysis of the contents of the intentions, perceptions, observation, and data registration was carried out through the triangulation of data and categorization of contents for issuing results. It was concluded that the use of this ICT enhanced the abilities of these students, confirming cognitive improvements for learning the topics of mathematical logic, communication and social sciences. It was also found that this tool allows a better and greater development of their social skills.

Keywords: ICT; Inclusive Education; Cognitive; Socio-emotional.

\section{Introducción}

Según la Unesco (2008), a partir de la Declaración de Salamanca en 1994, "las escuelas deben acoger a todos los niños, independientemente de sus condiciones físicas, intelectuales, sociales, emocionales, lingüísticas u otras. Deben acoger a niños discapacitados y niños bien dotados, a niños que viven en la calle y que trabajan, niños de poblaciones remotas o nómadas, niños de minorías lingüísticas, étnicas o culturales y niños de otros grupos o zonas desfavorecidos o marginados", (p.3).

Según Echeita y Ainscow (2011), "la escuela se hace responsable no solamente del progreso de los niños, sino que asume el deber de dar cuenta sus logros", (p.30). Esta declaración ha permitido que la evaluación de la experiencia de integración en el Perú sea cada vez más positiva en el sentido de motivar a las escuelas a abrir sus puertas y vencer la desconfianza de los maestros para atender la diversidad estudiantil (Reátegui et al, 2015). A la fecha se han registrado progresos y avances significativos en los procesos de socialización de los niños integrados, y un beneficio general para aquellos que, sin tener discapacidad, presentan dificultades de aprendizaje (Cueto, Rojas, Dammert \& Felipe, 2018). Se ha registrado, asimismo, una reducción de los prejuicios imperantes en la escuela y una mejora de las formas y cultura de convivencia (Boggio, 2018) y se ha tenido promisorias experiencias de enseñanza a estudiantes mediante las TIC (Jiménez, Serrano \& Prendes, 2017; Centeno \& Santillán, 2016; Cortés, 2015; Víquez, 2014). No obstante, aún no hay claridad sobre los logros de aprendizaje realmente obtenidos por los estudiantes incluidos sobre todo en Perú (Oyarce, 2016; Reátegui et al., 2015). 
En ese orden de ideas, el hecho de que un estudiante con necesidades educativas especiales (NEE) aprenda diferenciadamente, no imposibilita que los docentes le enseñen de la mejor manera posible y que la escuela implemente las adecuaciones favorables que permitan el desarrollo pleno de su potencial.

Así pues, la adopción de las TIC por parte de las entidades puede concebirse no como un importante reto, sino más bien como una importante oportunidad para los países centroamericanos, debido a sus aplicaciones sobre la capacidad de este sector de generar mayores oportunidades de empleo y riqueza, y combatir así la pobreza en estos países". (Monge, Alfaro \& Alfaro, 2005 p.20).

A partir de la publicación del Decreto Supremo $N^{\circ}$ 026-2003-ED, el Ministerio de Educación de Perú está implementando planes y proyectos que no solo garantizan la ejecución de acciones sobre educación, sino que se provea de una educación inclusiva. Este decreto resulta inspirador para llevar a cabo este proceso de transformación, enmarcado en los continuos cambios sociales generados por las numerosas innovaciones tecnológicas y, el crecimiento vertiginoso de las tecnologías de la información y de la comunicación, sirviendo de base para proyectos regionales que permiten incluir a los centros educativos especiales dentro de la educación básica regular, enfatizándose en la nueva denominación: "Centros de Educación Básica Especial" (CEBE) y el Programa de Intervención Temprana (PRITE). Lo positivo de esta transformación es que ya se ha iniciado en varias ciudades peruanas, sin embargo, la fragmentación sociocultural y el centralismo limita cubrir brechas sociales aún después de 15 años de emitida la política por el ministerio de educación, quedando la opción de los esfuerzos locales que brinden mayor fuerza y atención por parte de la sociedad, al servir de focos de experiencia positiva y posterior difusión a las áreas geográficas más alejadas del interior del país.

Bajo esta perspectiva, el uso de las tecnologías de la información y comunicación no solamente la convierte en una herramienta fundamental para el mejor entendimiento de la interacción académica en la educación básica, sino que también la vuelve una oportunidad para atender situaciones de discapacidad que contribuya a mejorar las condiciones de aprendizaje a los estudiantes dentro de las aulas. De esa manera, la propuesta de este estudio es conocer el impacto de las tecnologías de la información y la comunicación (TIC), como mecanismo innovador en su aplicación de las experiencias curriculares de los docentes, en el desarrollo de las habilidades cognitivas y socio emocionales en el contexto de un CEBE, pudiendo servir de modelo para otras instituciones educativas que asuman nuevos compromisos centrados en este tipo de estudiantes, proyectando nuevas alternativas de esperanza para la integración social y la igualdad de oportunidades mediante la participación oportuna de todos y todas; alcanzado mediante una educación innovadora, progresiva e inclusiva.

\section{La educación inclusiva.}

De acuerdo con Castillo, Sosa, Rapallini, Blanc y Lepratte (2013), se define como "una respuesta de enfoque filosófico, político, económico, social, cultural y pedagógico que persigue la valoración y aceptación de las diferencias de los alumnos dentro de la escuela" (p. 486). Con esta definición se hace necesario el re-planteamiento de las estrategias de las prácticas docentes, proponiendo innovaciones pedagógicas como "alternativas para la construcción de: respuestas a las necesidades educativas para las personas con barreras para el aprendizaje, su participación en distintos contextos, la promoción de las alfabetizaciones múltiples y el aprendizaje constructivo" (Zappalá, Köppel, \& Suchodolski, 2011). Por otra parte, con la intención de precisar, de acuerdo con lo vertido anteriormente, la 'educación inclusiva' subsumiría la concepción de 'educación especial', pues de acuerdo con Castillo, Sosa, Rapallini, Blanc, y Lepratte, (2013) ésta última se define como "una modalidad del sistema educativo orientada hacia el aseguramiento del derecho universal a la educación de aquellas personas con discapacidades, temporales o permanentes, en todos los niveles" (pp. 486-87). 
La inclusión de la educación especial en la definición es refrendada por la Unesco - Unicef (2001), cuando indicaron que la acepción de la educación inclusiva es más profunda, que el de integración. Para precisar, su concepción tiene un supuesto distinto, porque se encuentra conexo con la misma naturaleza de la educación básica regular y del significado de escuela. Refiere un modelo de escuela donde no hay "requisitos de ingreso" ni ningún otro procedimiento de selección. De acuerdo con este enfoque de educación, se debe "modificar substancialmente la Estructura, funcionamiento y propuesta pedagógica de las escuelas para dar respuesta a las necesidades educativas de todos y cada uno de los niños y niñas, de forma que todos tengan éxito en su aprendizaje y participen en igualdad de condiciones" (García, 2016, p. 5).

Cabe añadir como señala la UNESCO (2008) "El objetivo de la inclusión es brindar respuestas apropiadas al amplio espectro de necesidades de aprendizaje tanto en entornos formales como no formales de la educación más que un tema marginal que trata sobre cómo integrar a ciertos estudiantes a la enseñanza convencional, representa un enfoque que examina cómo transformar los sistemas educativos y otros entornos de aprendizaje, con el fin de responder a la diversidad de los estudiantes" (p.8).

\section{El uso de las Tics en la educación inclusiva.}

Vivimos en una sociedad que está inmersa en el desarrollo tecnológico, donde el avance de las TIC ha cambiado nuestra forma de vida, impactando en muchas áreas del conocimiento. En el área educativa, las Tics han demostrado que pueden ser de gran apoyo ya que favorecen la integración de colectivos con necesidades especiales (personas con dificultades motoras y sensoriales, intelectuales, entre otras), Monge, Alfaro y Alfaro (2005) pusieron en tapete la adopción de las TIC como una oportunidad que contribuiría en la mejora del aprendizaje de estudiantes con discapacidad, teniendo como refrendo la implementación de planes y mejoras por parte del Gobierno peruano a través del Ministerio de Educación.

Actualmente se ha logrado establecer un nexo que articula los principios de la educación mediante la construcción de nuevos conocimientos, y la pedagogía (confluencia de saberes) con el uso de las TIC en el sistema educativo. Ello significa una apertura bastante atractiva en el proceso de aprendizaje de los niños/as y adolescentes y, una oportunidad de enseñanza para los propios maestros. Así, el uso de las TIC, además de convertirse en una dinámica social, "contribuye en la implementación de cobertura, mejoramiento de la calidad educativa, y logros de mayor nivel en la descentralización, democracia y equidad" (D.S. N 067-2001-ED). De esta manera, se propicia en la educación que las TIC se conviertan en herramientas y no en un fin del proceso enseñanza y aprendizaje; sino que transforman estos procesos para apoyar y fomentar un modelo centrado en los estudiantes que requieren de asjutes razonables de acuerdo a sus necesidades educativas especiales.

Añadiendo a lo anterior, Bretel (2006) señaló que "el sistema educativo puede ser radicalmente distinto siempre y cuando se piense que la discapacidad sea un impedimento, dejando de lado, que las TIC no solo sea un medio del diluvio global; sino que la convierte en una tarea educativa" (p. 9). Esta idea se complementa con la propuesta de Somekh (cómo se citó en Parra, 2014, p. 23) cuando dijo que las TIC "deben integrarse en el proceso de enseñanza y aprendizaje, con el involucramiento de los docentes sobre pedagogía. De este modo, internet es la plataforma causal de encuentro multicultural de todos los tiempos".

\section{Categorías de análisis.}

El primer aspecto que se tuvo en consideración, fue la relevancia -tanto para los estudiantes como para los docentes - entendiéndose como acciones creadas en la plataforma virtual para el proceso de enseñanza-aprendizaje para escolares de primaria según los cursos correspondientes, programados en las unidades temáticas educativas. 
Un segundo aspecto a considerar fue la pertinencia del estudio. Aquello implicó haber realizado varias visitas al Centro de Educación Básica Especial; instalar un programa y módulos (aplicaciones) en el cual el estudiante lograra asociar una serie de características de las asignaturas, propias del sistema educativo oficial con las características peculiares de la población escolar a la cual estaba dirigida.

El tercer aspecto para considerar fue lo innovador como proyecto y como propuesta de gestión educativa. En efecto, las experiencias realizadas en otros países han servido como insumo para elaborar la propuesta de trabajo utilizando las TIC para personas con discapacidad, una alternativa que se maneja y se vive en aula muchas veces; puesto que todo proceso de innovación permite hacer ajustes a los contenidos de aprendizaje y las dinámicas y/o características de acuerdo con cada una de las necesidades especiales que presente cada estudiante buscando atender su discapacidad. Asimismo, se ha considerado que la propuesta comunicativa sea novedosa, puesto que las TIC, en la actualidad permite trabajar de manera innovadora, creativa y didáctica a los docentes.

Como último aspecto, se ha estipulado que la propuesta genere aprendizajes significativos en cada uno de los estudiantes del Centro de Educación Básica Especial. Al respecto se creó un software educativo, dicho programa fue instalado en las computadoras del laboratorio, de modo que cada estudiante tuvo una máquina; el docente fue formulando preguntas a cada estudiante para que cada uno de ellos vaya respondiendo y/o identificando elementos significativos.

\section{Método}

El estudio correspondió a una investigación cualitativa, mediante la investigación acción se logró identificar los hallazgos, luego se realizó el análisis de contenidos de las intenciones, percepciones, observación, registro de datos mediante la triangulación de datos y categorización de contenidos.

\section{Caracterización de sujetos.}

La aplicación del proyecto requirió de ocho personas, de 08 a 13 años que presentaron evidente discapacidad para hablar o de moverse y que formaban parte de la comunidad educativa del Centro de Educación Básica Especial "Corazón de Jesús" de la provincia de Jaén, Cajamarca. La intencionalidad del proyecto fue captar las reacciones de los estudiantes mediante el uso de las tecnologías en diferentes áreas pedagógicas como parte de sus cursos regulares de la enseñanza básica. Para ello, fue necesario acondicionar programas informáticos (softwares) y aplicarlos en algunas áreas bajo la dirección de los docentes.

\section{Herramienta empleada.}

Se empleó el software Plaphoons (Plaphoons v: 939.), es un sistema de comunicación para persones con discapacidad física (Programa que pertenece al Projecte Fressa). Se logró acondicionarlo a computadoras y tablets mediante aplicaciones diversas (SpaecePLAPHOONS v: 116; AndroidPlaphoons v: 78; RataPlaphoons v: 76; Kanghooru v: 117; Controlador de un Teclado; Pasa páginas; Mouse Joystick; Conversor Mouse Teclat; Conversor micrófono estéreo a mouse o teclado; JClicFressa v: 106 ; mouse; Texto a MP3; El Xerraire v: 38; Navegador Web Hablado y/o controlado por escaneo; Lectura de libros para invidentes; Globus 3 v: 142; Motor de reconocimiento de sonidos; Taules 2 . Reconocimiento de fonemas, Reconocimiento de vocales entre otros más). Es una herramienta de mucha utilidad para el aprendizaje de lectoescritura de dichas personas. Cuenta con un sistema operativo como si se tratase en cualquier procesador textual (Microsoft Word) pero al mismo tiempo como sistema de control a distintos buscadores de internet (Google Chrome, Internet explorer y Mozilla Firefox). 


\section{Resultados}

\section{El impacto del uso de las TIC en la educación básica especial del nivel primario.}

Al emplear las TICs en la enseñanza, el estudiante tuvo la oportunidad de potencializar sus capacidades comunicativas e intelectuales, desarrollar sus competencias y habilidades. Una vez instalado el programa, para el estudiante fue fácil seleccionar mediante un clic cualquiera de las ventanas que presenta el programa, las mismas le permitieron explorar nuevos campos de acción pedagógica. Con la interacción que realicen los estudiantes con la computadora, se observó que incrementó su familiaridad con las TIC; ello permite al docente emplear diferentes estrategias pedagógicas para el proceso de enseñanza y aprendizaje de su asignatura.

Se evidenció que el estudiante frente a la computadora también hizo uso de su pensamiento crítico y de su gestualización silábica. Al observar las imágenes identificaron escenas de la realidad virtual, dando asimismo sus propias respuestas; no obstante, la inferencia que hicieron al momento de conectarse con el mundo virtual, contrastó con los pre-supuestos del estudio: el desinterés de aprender mediante las TIC. Por el contrario, se notó mayor interés. El entusiasmo se prolongó más de la cuenta; por lo que se tuvo que seleccionar por parejas, y otorgar 20 minutos a cada estudiante, racionalizando el tiempo de la sesión de clase. Por consiguiente, explorar este nuevo campo de aprendizaje ha traído ventajas; por un lado, el descubrimiento de un mundo distinto al que viven a diario (con materiales educativos), traduciéndose así el proceso educativo en un logro pedagógico y por otro, la expectativa de la ampliación del conocimiento en sí.

Otro aspecto que se evidenció en esta categoría fue que permitió representar su propio mundo. Representar el mundo en que se vive para un niño con discapacidad leve o moderada, puede ser semejante o igual al mundo que vive un niño normal. Sin embargo, esta herramienta ha permitido profundizar la visión que los niños tienen de su realidad física. Ante ello, en la observación se evidenció que muchos niños que estudian en el CEBE evaluado no tienen computadoras en casa, sin embargo, se constató que acudieron a las cabinas de internet, y son capaces de navegar, algunos de ellos (los mayores, con discapacidad física) tienen facebook, y una red social, interactuando en tiempo real, dando lugar a una nueva identidad social mediante el mundo informático. En consecuencia, representar su propio mundo es ganar autonomía e independencia social.

\section{El uso de las TIC en habilidades cognitivas.}

Para ello, se ha consideró resultados de acuerdo con los siguientes criterios de análisis:

Didácticas de enseñanza y aprendizaje: Se relacionaron con la metodología de enseñanza, permitiendo de este modo redefinir el aprendizaje formativo, explicando y proponiendo orientaciones científicas para los problemas didácticos y el cambio docente, la educación del alumno de todos los niveles educativos y la formación pedagógica que se aplica al docente del CEBE evaluado. El software Plaphoons tiene un módulo o sub programa llamado SpaecePLAPHOONS que permite el control de otros programas o software y dinamiza el acercamiento entre la tecnología y la educación, frente a ello, los niños que participaron en el estudio descubrieron aspectos didácticos no vistos anteriormente, como el caso de construir bloques (rompecabezas o puzle, aplicaciones descargadas de internet previamente - Tangram) mediante la manipulación del mouse de pie y la observación de la pantalla.

En el área cognitiva de lógico-matemática: que tuvo en cuenta el razonamiento lógico matemático a partir del uso de las TIC. Al momento de evaluación la participación de los niños, se logró evidenciar, que los niños con discapacidad física y motora no tuvieron dificultades en la comprensión y resolución de problemas matemáticos, sin embargo, para aquellos niños que tiene 
discapacidad intelectual leve, se les hace más difícil resolver los problemas, el manejo de figuras geométricas es una sus actividades preferidas.

En el área de comunicación: se logró evidenciar que los niños lograron identificar colores, formas, lenguaje silábico, comprende textos orales, escucha primero el texto y luego comprende la lección, realiza la tarea. Se expresa oralmente o con señales o el lenguaje de señas para los estudiantes sordos, comprende textos escritos, que pueden ser traducidos por los encargados y ejecuta lo aprendido en la computadora. En esta área fue más fácil desarrollar actividades de comprensión lectora, pues se reforzó los textos con audio libros y cuentos descargados de internet.

\section{El uso de las TIC en habilidades socioemocionales.}

El estudio concentró varios elementos que repercutieron en las actividades de los estudiantes y docentes. Las subcategorías que se evidenciaron en estos hallazgos fueron:

En el Área de ciencias sociales: Tuvo en cuenta la aplicación de las ciencias sociales como enciclopedias y videos de históricos y a partir del uso de las TIC. Así se pudo observar que construyeron representaciones históricas a partir de interpretaciones que se proveyeron en las clases.

Habilidades sociales: Se pudo evidenciar la expresión de habilidades como el autocontrol de los estudiantes, permitió respetar su turno; paciencia para la resolución de problemas facilitando el logro de resolver etapas por sí solos y el apoyo hacia sus compañeros, demostraron solidaridad entre estudiantes y docentes, se establecieron normas de convivencia y reglamento para el aula de informática.

\section{Discusión}

Actualmente, el uso de las TIC se ha consolidado como un elemento fundamental, inherente al desarrollo humano, y desde luego la educación no ha escapado de ello, se explora un sin número de posibilidades para el desempeño educativo, expandiéndose cada vez más a otros espacios, de manera que el conocimiento se globaliza. "Cobra vital importancia su universalización, llegando a las esferas de la educación especial; su impacto no solo se centra en brindar información, también convertirse en un canal de comunicación, instrumento cognitivo y procesamiento del conocimiento" (Prieto et al, 2011, p. 95). En este sentido, el uso de las TIC en el CEBE se ha consolidado como una alternativa para la transmisión de conocimientos, y procesarlos en los niños de primaria; integrándose como una opción innovadora frente a la escuela veterana tradicional y excluyente (Juárez, Comboni \& Garnique, 2010; Castro, Castañeda, Ossa, Blanco, \& Castillo, 2017; López \& Valenzuela, 2015).

Frente a ello, el uso de las TIC, han motivado a que los alumnos logren habilidades cognitivas, interactúen con sus pares, y adquieran hábitos durante las didácticas de enseñanza y aprendizaje. De acuerdo a los hallazgos se evidenció que algunos docentes permitieron esta interacción virtual; pero también hubo docentes que, por el contrario, no exploraron esta herramienta porque evidenciaron desconocimiento del manejo de la misma, empero, ello no significó que fuesen reticentes hacia la inclusión de niños con necesidades educativas especiales (Castro et al., 2017, p. 106).

En cuanto a la experiencia del área cognitiva de lógico-matemático y de comunicación, se evidenció el hábil desempeño de los niños y niñas con discapacidad motora para la comprensión de texto, y/o resolución de problemas matemáticos y expresión comunicacional, aun cuando hubieron algunos con dificultades en sus expresiones orales (López y Valenzuela, 2015, p. 45); por otro lado, fue distinto la acción de los niños y niñas con discapacidad intelectual en quienes se evidenció mayores dificultades; puesto que no poseen un nivel suficiente de los procesos cognitivos (Gutiérrez, 2014); debido a que, a mayor complejidad de problemas, mayor 
exigencia requiere de los estudiantes para comprender y, de los docentes para enseñar. Hallazgo que concuerda con las habilidades de resolución de problemas, que involucra del pensamiento lógico y enunciado verbal que, en muchos casos, es detectado a temprana edad (Espinoza, Marco, \& Ygual, 2018).

En relación al uso de esta herramienta en las habilidades socioemocionales, los hallazgos más relevantes fueron similares a las de las otras áreas, con la diferencia que en ciencias sociales se construyeron representaciones sociales (Weisz, 2017).

Por otro lado, se evidenció que las habilidades sociales establecieron normas de convivencia, y reglamento para el aula de informática entre los niños y niñas, lo que sirve de apoyo para mejorar su comunicación e interacción social (Martínez \& Fernández, 2015).

En consecuencia, la relevancia de los hallazgos ha podido concatenarse con las propuestas de diversos autores que, de un modo y otro, se centran en la persona con necesidades educativas especiales, y no como aquella que se convierte en un obstáculo social, con una legislación que aún guarda vacíos para incluir educativamente a esta población (Padilla, 2011).

El empleo de esta herramienta abre un espacio promisorio para la potenciación de las áreas cognitivas en estudiantes de educación básica con discapacidad vocal y motora en quienes se constató mejoras cognitivas para el aprendizaje de los tópicos de lógico matemática, comunicación y ciencias sociales. Se evidenció también que el aprendizaje de las aplicaciones de la herramienta Plaphoons (software) permitió mejor y mayor desarrollo de las habilidades sociales de los estudiantes evaluados, facilitándoles la interacción social a través de redes virtuales tanto dentro de la Institución educativa como también en sus entornos familiares.

\section{Referencias}

Bretel, L. (2006). El diluvio Global. Computadoras e internet en la escuela. Revista de Educación. El Educador, 2(7), 4-9. Recuperado de https://www.MINEDUcacion.gov.co/cvn/1665/articles-129277_archivo_pdf.pdf

Boggio, M. S. (2018). Promoviendo estrategia de gestión para la atención pertinente en inclusión educativa: buena práctica. (Tesis de grado). Lima, Perú: Pontificia Universidad Católica del Perú. Recuperado de: http://tesis.pucp.edu.pe/repositorio/handle/123456789/10288

Castillo, E., Sosa, R., Rapallini, U., Blanc, R., \& Lepratte, L. (Octubre, 2013). TICs para una Educación Inclusiva. Presentado en el XIX Congreso Argentino de Ciencias de la Computación - CACIC - . XI Workshop Tecnología informática aplicada en Educación - WTIAE. Mar del Plata, Argentina. Recuperado de: https://bit.ly/2N33AG0

Castro-Rubilar, F., Castañeda-Díaz, M., Ossa-Cornejo, C., Blanco-Hadi, E. \& CastilloValenzuela, N. (2017). Validación de la escala de autoadscripción inclusiva en docentes secundarios de Chile. Psicología Educativa 23(2), 105-113. Doi: https://doi.org/10.1016/j.pse.2017.05.003

Centeno, H. D., \& Santillán, A. T. (2016). Sistema de comunicación móvil basado en la metodología aumentativa alternativa en realidad aumentada para niños con síndrome de Down. (Tesis de grado). Chimborazo, Ecuador: Escuela Superior Politécnica de Chimborazo. Recuperado de: https://docplayer.es/89429124-Escuela-superiorpolitecnica-de-chimborazo.html

Cortés J. (2015) Incorporación de las TIC como estrategia pedagógica para determinar las relaciones y procesos multiculturales en niños, niñas y jóvenes de séptimo grado de una institución educativa oficial del distrito capital. (Tesis para de Maestría). Bogotá: Universidad Distrital "Francisco José de Caldas". Recuperado de: http://repository.udistrital.edu.co/bitstream/11349/2118/1/Cort\%C3\%A9sPedrazaJulioR einaldo2015.pdf

Cueto, S., Rojas, V., Dammert, M., \& Felipe, C. (2018). Cobertura, oportunidades y percepciones sobre la educación inclusiva en el Perú. Lima, Perú: Ministerio de Educación. Recuperado de: https://bit.ly/2NvOSvA 
Echeita, G., \& Ainscow M. (2011). La educación inclusiva como derecho. Marco de referencia y pautas de acción para el desarrollo de una revolución pendiente. Tejuelo: Revista de Didáctica de la Lengua y la Literatura 12, 26 - 46. Recuperado de: http://hdl.handle.net/10486/661330

Espinoza, L., Marco, R., \& Ygual, A. (2018). Conciencia fonológica y resolución de problemas matemáticos en educación infantil. Rev. Logopedia, Foniatría y Audiología 38(2), 61-68. Doi: https://doi.org/10.1016/j.rlfa.2017.07.003

García, G. (2016). Las TIC en la Educación Inclusiva: Una estrategia pedagógica transformadora en jóvenes y adultos. México: Universidad Nacional Autónoma de México. Recuperado https://reposital.cuaed.unam.mx:8443/xmlui/handle/123456789/4730

Gutiérrez, R. (2014). La composición escrita de textos narrativos en alumnos sordos de educación secundaria. Aula Abierta 42(1), 22 - 27. Doi: https://doi.org/10.1016/S02102773(14)70004-6

Jiménez, M. D., Serrano, J. L., \& Prendes, M. P. (2017). Estudio de caso de la influencia del aprendizaje electrónico móvil en el desarrollo de la comunicación y el lenguaje con un niño con TEA. Educar, 53(2), 419-443. Doi: https://doi.org/10.5565/rev/educar.782

Juárez, J., Comboni, S., \& Garnique, F. (2010) De la educación especial a la educación inclusiva. $\begin{array}{lll}\text { Argumentos, } & \text { 23(62):41-83. Recuperado de }\end{array}$ http://www.scielo.org.mx/scielo.php?script=sci_arttext\&pid=S018757952010000100003

López I., \& Valenzuela, G. (2015) Niños y adolescentes con necesidades educativas especiales. Revista Médica Clínica Las Condes, 26(1), 42-51. Recuperado de https://doi.org/10.1016/j.rmclc.2015.02.004

Martínez, V., \& Fernández, J. (2015) Aplicación de un programa de educación psicomotriz en un caso con syndrome de Down. Magister 27(2), 67-75. Doi: 10.1016/j.magis.2015.12.003

Monge, R., Alfaro, C. \& Alfaro, J. L. (2005). TICs en Las PYMES de Centroamérica: Impacto de la Adopción de Las Tecnologías de la Información Y la Comunicación en El Desempeño de Las Empresas. Costa Rica: Editorial Tecnológica de Costa Rica.

Oyarce, M. J. (2016). Tecnologías de información y comunicación, TIC y su relación con el desempeño docente con calidad en la Escuela Académica Profesional de Comunicación Social de la Universidad Nacional Mayor de San Marcos, 2015. (Tesis de maestría). Lima, Perú: Universidad Nacional Mayor de San Marcos. Recuperado de http://cybertesis.unmsm.edu.pe/handle/cybertesis/4961

Padilla, A. (2011). Inclusión educativa de personas con discapacidad. Revista Colombiana de Psiquiatría, 40 (4), 670-699. Doi: https://doi.org/10.1016/S0034-7450(14)60157-8

Parra, K. N. (2014). El docente y el uso de la mediación en los procesos de enseñanza y aprendizaje. Revista de Investigación, 38(83), 155-180. Recuperado de http://www.scielo.org.ve/scielo.php?script=sci_arttext\&pid=S101029142014000300009

Prieto, V., Quiñones, I., Ramírez, G., Fuentes, Z., Labrada, T., Pérez, O., \& Montero, M. (2011). Impacto de las tecnologías de la información y las comunicaciones en la educación y nuevos paradigmas del enfoque educativo. Educación Médica Superior; 25 (1): 95-102. Recuperado de http://scielo.sld.cu/scielo.php?script=sci_arttext\&pid=S086421412011000100009

Reátegui, L. M., Izaguirre, M. H., Mori, H., Castro, R. S., \& Aguedo, N. (2015). Actitud de estudiantes y profesores del Departamento de Pediatría hacia las tecnologías de la información y comunicación (TICs). Anales de la Facultad de Medicina, 76(3), 261-264. Recuperado de: http://www.scielo.org.pe/scielo.php?script=sci_arttext\&pid=S1025$55832015000400007 \& \operatorname{lng}=\mathrm{es} \& \ln \mathrm{ln}=\mathrm{es}$

UNESCO (2008). Educación inclusiva: camino hacia el futuro. (48). Recuperado de: http://www.ibe.unesco.org/fileadmin/user_upload/Policy_Dialogue/48th_ICE/General_ Presentation-48CIE-4_Spanish_.pdf 
Unesco - Unicef (2001). Desafíos de la política educacional: Inclusión de niños con discapacidad en la escuela regular. Ciclo de debates (8). Recuperado de: http://www.unicef.cl/web/wpcontent/uploads/doc_wp/debate8.pdf

Víquez, I. G. (2014). Las TIC en la educación inclusiva e influencia en el aprendizaje de preescolares. Congreso Iberoamericano de Ciencia, Tecnología, Innovación y Educación. Buenos Aíres, Argentina. (Nov. 2014)

Weisz, C.B. (2017). La representación social como categoría teórica y estrategia metodológica. Revistas Científicas Universidad CES, 10(1), 99-108. Doi: http://dx.doi.org/10.21615/cesp.10.1.7

Zappalá, D., Köppel, A. \& Suchodolski, M. (2011). Inclusión de TIC en escuelas para alumnos con discapacidad intelectual. Buenos Aires, Argentina: Ministerio de Educación de la Nación. Recuperado de: https://bit.ly/2phkjfy 